\title{
ON EMBEDDING SET FUNCTIONS INTO COVARIANCE FUNCTIONS(1)
}

\author{
BY
}

\author{
G. D. ALLEN
}

\begin{abstract}
We consider any continuous hermitian kernel $M\left(\Delta, \Delta^{\prime}\right)$ on $\mathscr{P} \times \mathcal{P}$ where $\mathcal{P}$ is the prering of intervals of $[0,1]$. Conditions on $M$ are given to find an interval covariance function $K\left(\Delta, \Delta^{\prime}\right)$ so that $K\left(\Delta, \Delta^{\prime}\right)=M\left(\Delta, \Delta^{\prime}\right)$ for all nonoverlapping $\Delta$ and $\Delta^{\prime}$ in $\mathcal{P}$. The problem is solved by first treating finite hermitian matrices $A$ and finding a positive definite matrix $B$ so that $b_{i j}=a_{i j}, i \neq j$, so that tr $B$ is minimized. Using natural correspondence between interval covariance functions and stochastic processes, a decomposition theorem is derived for stochastic processes of bounded quadratic variation into an orthogonal process and a process having minimal quadratic variation.
\end{abstract}

1. Introduction. By a well-known theorem, there is a correspondence between second order stochastic processes and hermitian functions of two variables, of the nonnegative type (i.e. covariance functions). It is easily shown that a similar correspondence holds for the nonnegative definite functions of two variables and nonnegative biadditive functions of intervals (also called covariance functions). In this paper we consider hermitian biadditive functions of intervals, which are additionally of bounded variation, and discuss when they can be embedded into covariance functions, also of bounded variation. The study of this problem leads to a decomposition theorem for a class of stochastic processes of bounded quadratic variation. This paper also provides a step toward the study of nonorthogonally scattered measures.

2. Definitions and preliminary results. Throughout this paper all stochastic processes $X(t)$ are of the second order, with $t$ ranging in $[0,1]$.

If $\Delta^{\prime}$ and $\Delta^{\prime \prime}$ are two subintervals of $[0,1]$ which are adjacent but not overlapping, we write $\Delta=\Delta^{\prime}+\Delta^{\prime \prime}$ to be the smallest subinterval of $[0,1]$ containing $\Delta^{\prime}$ and $\Delta^{\prime \prime}$. If the expression $\Delta=\Delta^{\prime}+\Delta^{\prime \prime}$ is written it is assumed that $\Delta^{\prime}$ and $\Delta^{\prime \prime}$ are adjacent but not overlapping. Similarly if $\Delta=\sum_{i=1}^{n} \Delta_{i}$ it is assumed that $\Delta_{j}$ and $\sum_{i=1}^{j-1} \Delta_{i}$, for each $j \leq n$, are adjacent but not overlapping.

By a hermitian biadditive function of intervals we mean a function $K\left(\Delta, \Delta^{\prime}\right)$ of two arbitrary subintervals of $[0,1]$, such that

$$
K\left(\Delta, \Delta^{\prime}\right)=\overline{K\left(\Delta^{\prime}, \Delta\right)},
$$

and if $\Delta=\Delta_{1}+\Delta_{2}$ then $K\left(\Delta, \Delta^{\prime}\right)=K\left(\Delta_{1}, \Delta^{\prime}\right)+K\left(\Delta_{2}, \Delta^{\prime}\right)$ for all $\Delta^{\prime}$ in $[0,1]$. In all

Received by the editors November 10, 1971.

AMS (MOS) subject classifications (1970). Primary 60G05.

Key words and phrases. Covariance functions, bounded quadratic variation, decomposition of stochastic process.

(1) This paper includes part of the author's dissertation at the University of Wisconsin. The author expresses appreciation for the patience and help of his advisor, L. C. Young. 
that follows such a function will, for brevity, be called an interval function.

A partition $\supset$ of $[0,1]$ is a decomposition of $[0,1]$ into a finite number of nonoverlapping subintervals $\Delta_{1}, \ldots, \Delta_{n}$. If $\beth^{\prime}=\Delta_{1}^{\prime}, \ldots, \Delta_{m}^{\prime}$ is another partition of $[0,1]$ and for each $i, \Delta_{i}^{\prime} \subset \Delta_{j}$ for some $j$, we say $\beth^{\prime}$ is a refinement of $\supset$ and write $\beth^{\prime} \leq \beth$. Any partition $\sqsupset$ and interval function $K$ generates a matrix $\left(K\left(\Delta_{i}, \Delta_{j}\right)\right), i, j=1, \ldots, n$. We define a special sequence of partitions $\left\{\beth_{n}\right\}$ to be the binary partitions when $\beth_{n}$ is composed of $2^{n}$ subintervals having points of division the numbers $k / 2^{n}, k=0,1, \ldots, 2^{n}$. The elements of $\beth_{n}$, from left to right, are denoted by $\Delta_{i}^{n}, i=1, \ldots, 2^{n}$.

If for every partition $\beth=\Delta_{1}, \ldots, \Delta_{n}$ the matrix $\left(K\left(\Delta_{i}, \Delta_{j}\right)\right)$ is positive semidefinite then we say $K$ is of the nonnegative type. If $K(\Delta, \Delta) \geq 0$ for every $\Delta \subset[0,1]$ we say $K$ is nonnegative.

Consider any stochastic process $X(t)$. For any subinterval $\Delta=\left(t_{0}, t_{1}\right)$ we define $\Delta X=X\left(t_{1}\right)-X\left(t_{0}\right)$. For any two intervals $\Delta, \Delta^{\prime}$ of $[0,1]$ we define the interval function

$$
M\left(\Delta, \Delta^{\prime}\right)=E\left(\Delta X, \overline{\Delta^{\prime} X}\right)
$$

$M\left(\Delta, \Delta^{\prime}\right)$ is called the interval covariance function of $X$. We now prove the

Theorem 2.1. If $X(t)$ is a stochastic process, the covariance function $M\left(\Delta, \Delta^{\prime}\right)$ is an interval function of the nonnegative type. Conversely, if $K\left(\Delta, \Delta^{\prime}\right)$ is an interval function of the nonnegative type, then $K$ is the interval covariance function of some stochastic process.

Suppose we have a hermitian function $\Gamma\left(t, t^{\prime}\right)$ of two variables. If for every finite sequence $t_{1}<t_{2}<\ldots<t_{n}$ the matrix $\Gamma\left(t_{i}, t_{j}\right)$ is positive semidefinite we say $\Gamma$ is of the nonnegative type. If $X(t)$ is a stochastic process, the function $\Lambda\left(t, t^{\prime}\right)=\left(X(t), X\left(t^{\prime}\right)\right)$ is also called a covariance function. Our theorem is then analogous to the following classical result ([1], [2]):

Theorem 2.1'. In order that the hermitian function $\Gamma\left(t, t^{\prime}\right)$ be a covariance function it is necessary and sufficient that it be of the nonnegative type.

As Theorem $2.1^{\prime}$ is well known we shall demonstrate the equivalence of the two theorems. That is, we shall prove the

Lemma 2.2. If $\Gamma\left(t, t^{\prime}\right)$ is a covariance function on $[0,1] \times[0,1]$, then it defines an interval function $K(\Delta, \Delta)$ of the nonnegative type. Conversely, if $K$ is an interval covariance function it defines a function $\Gamma\left(t, t^{\prime}\right)$ of the nonnegative type.

Proof. The first statement is trivial, so we pass on to the converse. That is, $K\left(\Delta, \Delta^{\prime}\right)$ is a covariance function. For $t_{1} \leq t_{2}$, define $\Delta_{1}=\left(0, t_{1}\right)$ and $\Delta_{2}=\left(t_{1}, t_{2}\right)$. Then define 


$$
\begin{gathered}
\Lambda(0,0)=\Lambda(0, t)=\Lambda(t, 0)=0 \\
\Lambda\left(t_{1}, t_{2}\right)=K\left(\Delta_{1}, \Delta_{1}\right)+K\left(\Delta_{1}, \Delta_{2}\right) \\
\Lambda\left(t_{2}, t_{1}\right)=K\left(\Delta_{1}, \Delta_{1}\right)+K\left(\Delta_{2}, \Delta_{1}\right) .
\end{gathered}
$$

The function $\Lambda$ is well defined and hermitian. It remains to show that $\Lambda$ is of the nonnegative type. Let $t_{1} \leq t_{2} \leq \ldots \leq t_{n}$ be any numbers in $[0,1]$ and $\rho_{i}$, $i=1, \ldots, n$, any set of complex numbers. Define

$$
\Delta_{1}=\left(0, t_{1}\right), \Delta_{2}=\left(t_{1}, t_{2}\right), \ldots, \Delta_{n}=\left(t_{n-1}, t_{n}\right) .
$$

Then, using biadditivity of $K$, if $t_{i} \leq t_{j}$,

$$
\Lambda\left(t_{1}, t_{j}\right)=\sum_{k=1}^{i} K\left(\Delta_{k}, \Delta_{k}\right)+\sum_{k=1}^{i} \sum_{l=1}^{j} K\left(\Delta_{k}, \Delta_{l}\right)
$$

and

$$
\sum_{i, j=1}^{n} \Lambda\left(t_{i}, t_{j}\right) \rho_{i} \bar{\rho}_{j}=\sum_{j=1}^{n} \sum_{i=1}^{n}\left\{\sum_{k=1}^{i} K\left(\Delta_{k}, \Delta_{k}\right)+\sum_{k=1}^{i} \sum_{l=1}^{j} K\left(\Delta_{k}, \Delta_{l}\right)\right\} \rho_{i} \bar{\rho}_{j}
$$

Collecting terms, we find the coefficient of $K\left(\Delta_{m}, \Delta_{m}\right)$ is $\sum_{i, j \geq m} \rho_{i} \bar{\rho}_{j}$ and the coefficient of $K\left(\Delta_{k}, \Delta_{l}\right)$ is $\sum_{i=k}^{n} \sum_{j=l}^{n} \rho_{i} \bar{\rho}_{j}$. Defining $\sigma_{k}=\sum_{i=k}^{n} \rho_{i}$ we can rewrite (2.3) as

$$
\sum_{i, j=1}^{n} K\left(\Delta_{i}, \Delta_{j}\right) \sigma_{i} \bar{\sigma}_{j}
$$

and this quantity is nonnegative. Thus the lemma is established. By applying Theorem $2.1^{\prime}$ to $\Lambda\left(t_{1}, t_{2}\right)$ we establish Theorem 2.1 .

We say that an interval function $K$ can be embedded into an interval covariance function if there exists a covariance function $M$ such that whenever $\Delta$ and $\Delta^{\prime}$ are nonoverlapping subintervals, $K\left(\Delta, \Delta^{\prime}\right)=M\left(\Delta, \Delta^{\prime}\right)$.

An interval function $K$ is said to be of bounded variation if

$$
\sup \sum_{\Delta \in \supset}|K(\Delta, \Delta)|<\infty
$$

where the supremum is taken over all finite partitions of $[0,1]$. A stochastic process is said to be of bounded quadratic variation if its covariance is of bounded variation. We define the quadratic measure $w(t)$ of $K\left(\Delta, \Delta^{\prime}\right)$ as the lim sup of the sum $\sum_{\Delta \in \beth_{t}} K(\Delta, \Delta)$ over partitions $\beth_{t}$ of $(0, t)$, as the norm of $\beth_{t}$ tends to zero. Similarly we define the quadratic measure of a stochastic process as the quadratic measure of its interval covariance.

3. The algorithm and main results. In this section we answer the question as to when a nonnegative interval function of bounded variation can be embedded into a covariance function of bounded variation. This question necessitates the study of the effect of the values of the interval function for nonoverlapping 
intervals on the values for coincident ones. Since by Theorem 2.1 we need consider only finite partitions and hence finite matrices, we are really asking what is the effect of the off diagonal terms on the diagonal terms. More precisely, if $A=\left(a_{i j}\right)$ is an $n$th order hermitian matrix with $a_{i i}=0$, what diagonal matrix $D$ must be provided in order to make $A+D$ positive semidefinite? In fact, what is the smallest (in some sense) $D$ that can be added? For a nonnegative interval function and a partition $\supset=\Delta_{1}, \ldots, \Delta_{n}$, the quantity for computing the variation is $\sum_{i=1}^{n} K\left(\Delta_{i}, \Delta_{i}\right)$, which is the trace of the matrix $\left(K\left(\Delta_{i}, \Delta_{j}\right)\right), i, j$ $=1, \ldots, n$. Thus it is the trace which is to be as small as possible for our problem. We will consider for the time being only matrices.

Let $A$ be an $n$th order hermitian matrix with zeros on the diagonal. Let $D^{\prime}$ be a diagonal matrix, and set $A^{\prime}=A+D^{\prime}$. We say that $A^{\prime}$ is a minimal positive semidefinite matrix generated by $A$ if (i) $A^{\prime}$ is positive semidefinite, and (ii) if $A^{\prime \prime}=A+D^{\prime \prime}$, where $D^{\prime \prime}$ is a diagonal matrix, then $\operatorname{tr} \Delta^{\prime} \leq \operatorname{tr} \Delta^{\prime \prime}$. Note that there is no guarantee of unicity. We intend to construct a matrix $D$ so that $A+D$ will be a minimal positive semidefinite matrix.

To that end, suppose that $A$ is as above. Let $\left\{x_{i}\right\}$ be a dense set of vectors on the unit sphere of $C^{n}$. In coordinates $x_{i}$ is given by $\left(x_{i 1}, x_{i 2}, \ldots, x_{i n}\right)$. Consider the following set of problems, $P_{m}$ :

Find real numbers $a_{i}^{m}, i=1, \ldots, n$, such that

$P_{m} \quad \mathcal{A}^{m}=\sum_{i=1}^{n} a_{i}^{m}$ is a minimum, subject to

$$
\sum a_{i}^{m}\left|x_{k i}\right|^{2} \geq-\left(A x_{k}, x_{k}\right), k=1,2, \ldots, m \text {, and } a_{i}^{m} \geq 0 \text {. }
$$

Let $\lambda_{i}, i=1, \ldots, n$ be the eigenvalues of $A$. Then each problem $P_{m}$ is a linear programming problem with a feasible solution, namely $a_{i}^{m}=d$ where $d$ $=\max _{1 \leq i \leq n}\left(-\lambda_{i}\right)$. This is clear, for then

$$
\sum_{i=1}^{n} a_{i}^{m}\left|x_{k i}\right|^{2}=d
$$

for every unit vector, and

$$
\inf _{\|x\|=1}-(A x, x)=d
$$

Thus, we may assume $\sum a_{i}^{m} \leq n d$, a uniform bound for all $m$. It is easy to see that $\mathscr{A}^{m} \leq \mathcal{A}^{m+1}$, since the difference is due to one additional constraint. This, together with $\sum a_{i}^{m} \leq n d$, implies that lim $\mathcal{A}^{m}=\mathcal{A}$ exists and is bounded. Now extract a subsequence $\left\{m^{\prime}\right\}$ so that $\left\{a_{i}^{m^{\prime}}\right\}$ converges for each $i$; call the limits $\left\{a_{i}\right\}$, $i=1, \ldots, n$. Define

$$
A^{\prime}=A+\operatorname{diag}\left(a_{1}, \ldots, a_{n}\right)
$$


The matrix is clearly positive semidefinite. That it has minimal trace is clear from construction.

Using a standard result from the theory of linear programming we have that the solutions for each $P_{m}$ form a closed, bounded, convex set, $C_{m}$ in $R_{n}$. Also $C_{m} \supseteq C_{m+1}$. Thus the set of solutions to the limit problem is just $\cap C_{m}=C$, which is also convex. Since $\operatorname{tr} A_{m}=0, C$ is separated from the origin in $R_{n}$. Thus in the euclidean norm on $R^{n}$ there is a unique element of minimal norm, still called $\left(a_{1}, \ldots, a_{n}\right)$. We call the matrix $A^{\prime}$ determined from this element the minimal positive semidefinite matrix generated by $A$. It is not known if $A^{\prime}$ can be obtained in a finite number of steps.

To recapitulate, our algorithm is the following sequence of steps: (i) Consider the hermitian matrix $A-\operatorname{diag}(A)$; (ii) find using the above procedure the positive semidefinite matrix $A^{\prime}$ so that $A-\operatorname{diag} A=A^{\prime}-\operatorname{diag} A^{\prime}$ in such a way that if another positive semidefinite matrix $A^{\prime \prime}$ satisfying $A-\operatorname{diag} A=A^{\prime \prime}-\operatorname{diag} A^{\prime \prime}$ is exhibited then $\operatorname{tr} A^{\prime} \leq \operatorname{tr} A^{\prime \prime}$; (iii) among all $A^{\prime}=\left(a_{i j}^{\prime}\right)$ found in step (ii) select that one satisfying $\sum_{i=1}^{n}\left(a_{i i}^{\prime}\right)^{2}=$ minimum. This matrix $A^{\prime}$ is called the minimal positive semidefinite matrix generated by $A$.

Before applying the algorithm to interval functions we make the following remarks:

(1) If $A^{\prime}$ is as constructed in the algorithm then it has at least one zero eigenvalue.

(2) If $A(t)$ is continuous in $t, t$ in a metric space, then $A^{\prime}(t)$ is continuous. $(A(t)$ is a symmetric $n \times n$ matrix function of $t$.)

(3) If $\lambda_{1}, \lambda_{2}, \ldots, \lambda_{n}$ are the eigenvalues of $A$ and $a_{1}, \ldots, a_{n}$ the diagonal elements of $A^{\prime}$, then $\sum\left|\lambda_{i}\right| \leq 2 \sum a_{i}$.

The proofs of (1) and (3) are elementary so we prove (2). Using a known result from linear programming the set-valued functions $C_{m}(t)$ are continuous in $t$, and, in fact, because $S$ is compact, the $C_{m}(t)$ are uniformly continuous in $t$. As in the case where $A$ is constant, we have $C_{m+1}(t) \subset C_{m}(t)$ for each $t$ and $m$. We define $C(t)=\bigcap_{m} C_{m}(t)$. We wish to show that $C(t)$ is continuous, that is, for any $\varepsilon>0$ there is a $\rho>0$ so that $\delta\left(C(t), C\left(t^{\prime}\right)\right)<\varepsilon$ if $d\left(t, t^{\prime}\right)<\rho$. Here $\delta(\cdot, \cdot)$ is the usual Hausdorff metric, and $d(\cdot, \cdot)$ is the metric on $S$. Also, we know that for every $\varepsilon^{\prime}>0$ there is an $M$ and a $\rho^{\prime}>0, \delta\left(C_{m}(t), C(t)\right)<\varepsilon^{\prime}$ and $\delta\left(C_{m}(t), C_{m}\left(t^{\prime}\right)\right)<\varepsilon^{\prime}$ if $m>M$ and $d\left(t, t^{\prime}\right)<\rho^{\prime}$. Application of the triangle inequality gives

$$
\delta\left(C(t), C\left(t^{\prime}\right)\right) \leq \delta\left(C(t), C_{m}(t)\right)+\delta\left(C_{m}(t), C_{m}\left(t^{\prime}\right)\right)+\delta\left(C_{m}\left(t^{\prime}\right), C_{m}(t)\right)<\varepsilon
$$

if we take $\varepsilon^{\prime}=\varepsilon / 3$. This proves the continuity of $C(t)$.

Now, as in the case of constant $A$, we define $a(t)=\left(a_{1}(t), \ldots, a_{n}(t)\right)$ to be that vector in $C(t)$ which is closest to the origin (in $R^{n}$ ). Since $C(t)$ is closed, convex, and disjoint from the origin, existence of $a(t)$ is assured. That this selection is continuous is known.

We turn now to nonnegative interval functions and the original question of this 
section. Let $\supset$ be a partition of $[0,1]$ into $n$ subintervals $\Delta_{1}, \Delta_{2}, \ldots, \Delta_{n}$. Consider the $n$th order matrix $A=\left(a_{i j}\right)$ defined by

$$
\begin{aligned}
a_{i j} & =K\left(\Delta_{i}, \Delta_{j}\right), & & i \neq j, \\
& =0, & & i=j .
\end{aligned}
$$

Let $A^{\prime}$ be the minimal positive definite matrix generated by $A$. Now let $\left\{\beth_{n}\right\}$ be any sequence of partitions, $\beth_{n}=\Delta_{1}^{n}, \Delta_{2}^{n}, \ldots, \Delta_{m(n)}^{n}$, with $\beth_{n} \leq \beth_{n-1}$, and $\lim \left|\beth_{n}\right|$ $=0$. Then each $\beth_{n}$ generates a matrix $A_{n}$, and $A_{n}$ generates $A_{n}^{\prime}$, by the algorithm.

If $\lim \operatorname{tr} A_{n}^{\prime}<M<\infty$, we say $K$ satisfies a $\mathrm{PD}$ condition relative to $\left\{\beth_{n}\right\}$. If $\lim \operatorname{tr} A_{n}^{\prime}<M<\infty$ for every such sequence $\left\{\beth_{n}\right\}$ and $M$ is fixed, we say that $K$ satisfies a PD condition. If when $\Delta_{1} \supset \Delta_{2} \supset \ldots$ and $\cap \Delta_{i}$ is a point

$$
\lim _{i} \sum_{\Delta_{j}^{n} \subset \Delta_{i}} a_{j}^{n}=0
$$

we say that $K$ satisfies a zero $P D$ condition.

In the following results we impose two conditions on the interval function $K\left(\Delta, \Delta^{\prime}\right)$ : (wl) the quadratic measure $w_{k}(t)$ it generates is continuous, and (w2) the quadratic measure $w_{k}(t)$ can be generated by any decreasing sequence of partitions $\beth_{n}^{t}$, of $[0, t]$ with $\lim \left|\beth_{n}^{t}\right|=0$. With these conditions we can prove the

Lemma 3.1. If $w_{k}(t)$ is as above and if $\left\{\beth_{n}\right\}$ is a decreasing sequence of partitions such that $\lim \left|\beth_{n}\right|=0$, then for every integer $p \geq 0$,

$$
\lim _{n \rightarrow \infty} \sum_{\Delta \in \supset_{n}} \sum_{\Delta^{\prime}, \Delta^{\prime \prime} \in \supset_{n+p} ; \Delta^{\prime}, \Delta^{\prime \prime} \subset \Delta ; \Delta^{\prime} \neq \Delta^{\prime \prime}} K\left(\Delta^{\prime}, \Delta^{\prime \prime}\right)=0 .
$$

Also, if $\square$ is any partition of $\Delta$

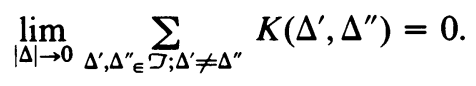

Proof. We know that

$$
\lim _{\Delta \in \supset_{n}} K(\Delta, \Delta)=\lim \sum_{\Delta \in \supset_{n+p}} K(\Delta, \Delta)
$$

and the quantity (3.2) is the difference

$$
\sum_{\Delta \in \supset_{n}} K(\Delta, \Delta)-\sum_{\Delta \in \supset_{n+p}} K(\Delta, \Delta)
$$

This gives the result. The second limit is an immediate consequence of the conditions (w1) and (w2) also.

Lemma 3.3. If $X(t)$ is a stochastic process with covariance function $K\left(\Delta, \Delta^{\prime}\right)$, and if the quadratic mecsure $w_{k}(t)$ satisfies the conditions (w1) and (w2), then $K\left(\Delta, \Delta^{\prime}\right)$ satisfies a zero PD condition. 
Proof. Let $\supset$ be any partition of $[0,1]$ with small norm. Then

$$
w(1) \doteq \sum_{\Delta \in \supset}(\Delta X, \Delta X)
$$

and further by the continuity of $w(t)$ each $(\Delta X, \Delta X)$ is small. As $|\supset| \rightarrow 0$,

$$
\sum_{\Delta \in \supset}(\Delta X, \Delta X)^{2} \rightarrow 0 .
$$

Now application of the algorithm to the covariance $K\left(\Delta, \Delta^{\prime}\right)$ of $X(t)$ gives that for each $\supset$ the constructed matrix must be dominated by (wl). And also, the sum of the diagonal elements squared must be dominated by

$$
\sum_{\Delta \in \supset}(\Delta X, \Delta X)^{2}
$$

This gives the lemma.

Before stating the main results we prove

Lemma 3.4. Suppose $A$ is an nth order hermitian positive semidefinite matrix, and suppose $0=q_{0}<q_{1}<\ldots<q_{p}=m$ are integers. If we define

$$
b_{i j}=\sum_{l=q_{i-1}+1}^{q_{i}} \sum_{m=q_{j-1}+1}^{q_{j}} a_{l m},
$$

the pth order matrix $B=\left(b_{i j}\right)$ is also hermitian and positive semidefinite.

Proof. Consider any vector $x \in R^{p}$. Then $x$ can be embedded into $R^{m}$ in the following way:

$$
x=\left(x_{1} \ldots x_{p}\right) \rightarrow y=\left(x_{1} \ldots x_{1}, x_{2} \ldots x_{2}, \ldots, x_{p} \ldots x_{p}\right)
$$

where $x_{i}$ is repeated $r_{i}$ times and $r_{i}=q_{i}-q_{i-1}$. It is clear that $(B x, x)=(A y, y)$, and the lemma is proved.

In the following theorem $K\left(\Delta, \Delta^{\prime}\right)$ satisfies the conditions (w1) and (w2)

Theorem 3.5. If $K\left(\Delta, \Delta^{\prime}\right)$ is a continuous nonnegative interval function of bounded variation that satisfies a zero PD condition, then $K$ can be embedded in a continuous interval covariance function, also of bounded variation.

Proof. We intend to construct a covariance function $M\left(\Delta, \Delta^{\prime}\right)$ for all $\Delta, \Delta^{\prime}$. First, define $M\left(\Delta, \Delta^{\prime}\right)=K\left(\Delta, \Delta^{\prime}\right)$ for all $\Delta$ and $\Delta^{\prime}$ nonoverlapping.

To define $M$ on coincident intervals we consider the subsequence $\beth_{n}$ of the binary partitions. As described above each $\beth_{n}$ describes via $K$ a matrix $A_{n}$, for which $A_{n}^{\prime}$ is the minimal positive definite matrix generated by $A_{n}$. Then substituting the diagonal elements of $A_{n}^{\prime}$ for those of $K\left(\Delta_{i}^{n}, \Delta_{j}^{n}\right)$ we arrive at an interval function $K_{n}\left(\Delta, \Delta^{\prime}\right)$, defined for all $\Delta$ and $\Delta^{\prime}$ which are intervals or sums of intervals in $\beth_{n}$. In fact for these it is biadditive and if $\mathcal{P}=\left(\pi_{1} \ldots \pi_{m}\right)$ is any 
partition of $[0,1]$ having intervals with endpoints contained by those of $\beth_{n}$, the matrix $K_{n}\left(\pi_{i}, \pi_{j}\right), 1 \leq i, j \leq m$, is positive semidefinite by Lemma 3.4. To complete the definition, define $K_{n}(\Delta, \Delta)=0$ if $\Delta$ is not of the form above, and define $K_{n}\left(\Delta, \Delta^{\prime}\right)=K\left(\Delta, \Delta^{\prime}\right)$ if $\Delta$ and $\Delta^{\prime}$ are not overlapping.

Let $S=\left\{\Delta_{1}, \Delta_{2}, \ldots\right\}$ be an enumeration of all the intervals of all $\beth_{n}$ in nonincreasing size. Then any $\Delta \subset[0,1]$ can be written either as an element of $S$, as a finite sum of elements of $S$, or as a limit of finite sums of elements of $S$.

We now define $M(\Delta, \Delta)$ for $\Delta \in S . K_{n}\left(\Delta_{i}, \Delta_{i}\right)$ exists for all $n$ and is bounded, because of the PD condition. Define $M\left(\Delta_{i}, \Delta_{i}\right)=\lim K_{n}\left(\Delta_{i}, \Delta_{i}\right)$. This defines $M(\Delta, \Delta)$ for all $\Delta \in S$. With these values we define $M(\Delta, \Delta)$ for all other $\Delta \subset[0,1]$.

Suppose now $\Delta \in S$ and $\Delta \subset \Delta^{\prime}$, so that $\Delta^{\prime}=\Delta+\Delta_{1}+\Delta_{2}$, each pair of $\Delta, \Delta_{1}$, $\Delta_{2}$ nonoverlapping; define

$$
\begin{aligned}
M\left(\Delta, \Delta^{\prime}\right) & =M(\Delta, \Delta)+M\left(\Delta, \Delta_{1}\right)+M\left(\Delta, \Delta_{2}\right) \\
& =M(\Delta, \Delta)+K\left(\Delta, \Delta_{1}\right)+K\left(\Delta, \Delta_{2}\right) .
\end{aligned}
$$

If $\Delta, \Delta^{\prime}$, and $\Delta^{\prime \prime}$ are in $S$ so that $\Delta=\Delta^{\prime}+\Delta^{\prime \prime}$ we must show that

$$
M(\Delta, \Delta)=M\left(\Delta^{\prime}, \Delta^{\prime}\right)+M\left(\Delta^{\prime \prime}, \Delta^{\prime \prime}\right)+2 \operatorname{Re} M\left(\Delta^{\prime}, \Delta^{\prime \prime}\right) .
$$

We know that, for any $\delta \in S$,

$$
M(\delta, \delta)=\lim K_{n}(\delta, \delta)
$$

and for $N$ large enough, $n>N$ implies

$$
K_{n}(\Delta, \Delta)=K_{n}\left(\Delta^{\prime}, \Delta^{\prime}\right)+K_{n}\left(\Delta^{\prime \prime}, \Delta^{\prime \prime}\right)+2 \operatorname{Re} K\left(\Delta^{\prime}, \Delta^{\prime \prime}\right)
$$

Taking the limit gives the desired equality. For finite sums of $\Delta \in S$, say $\Delta=\sum_{i=1}^{n} \Delta_{i}^{\prime}$, we also have

$$
M(\Delta, \Delta)=\sum_{i=1}^{n} M\left(\Delta_{i}^{\prime}, \Delta_{i}^{\prime}\right)+\sum_{i \neq j} M\left(\Delta_{i}^{\prime}, \Delta_{j}^{\prime}\right)
$$

Furthermore, if $\Delta=\sum_{i=1}^{m} \Delta_{i}^{\prime \prime}$ is another representation of $\Delta$ it is clear, by taking a common refinement of the two partitions, that

$$
M(\Delta, \Delta)=\sum_{i=1}^{m} M\left(\Delta_{i}^{\prime \prime}, \Delta_{i}^{\prime \prime}\right)+\sum_{i \neq j} M\left(\Delta_{i}^{\prime \prime}, \Delta_{j}^{\prime \prime}\right)
$$

What remains is to define $M(\Delta, \Delta)$ for all other $\Delta$. So, let $\Delta \subset[0,1]$. Then $\Delta$ can be written as a limit of finite sums $\delta_{n}=\sum_{i=1}^{n} \Delta_{i}^{\prime}$ of $\Delta_{i}^{\prime} \in S$. We define

$$
M(\Delta, \Delta)=\lim _{n \rightarrow \infty} M\left(\delta_{n}, \delta_{n}\right)
$$


To show this limit exists we note that

$$
K\left(\delta_{n}, \delta_{n}\right)=\sum_{i=1}^{n} K\left(\Delta_{i}^{\prime}, \Delta_{i}^{\prime}\right)+\sum_{i \neq j} K\left(\Delta_{i}^{\prime}, \Delta_{j}^{\prime}\right) .
$$

By first the continuity and second the nonnegativity of $K$ the following two limits exist:

$$
\lim _{n \rightarrow \infty} K\left(\delta_{n}, \delta_{n}\right)=K(\Delta, \Delta), \quad \lim _{n \rightarrow \infty} \sum_{i=1}^{n} K\left(\Delta_{i}^{\prime}, \Delta_{i}^{\prime}\right) .
$$

Therefore, $\lim _{n \rightarrow \infty} \sum_{i \neq j}^{n} K\left(\Delta_{i}, \Delta_{j}\right)$ exists. Now

$$
\begin{aligned}
\lim _{n \rightarrow \infty} M\left(\delta_{n}, \delta_{n}\right) & =\lim _{n \rightarrow \infty}\left(\sum_{i=1}^{n} M\left(\Delta_{i}^{\prime}, \Delta_{i}^{\prime}\right)+\sum_{i \neq j} M\left(\Delta_{i}^{\prime}, \Delta_{j}^{\prime}\right)\right) \\
& =\lim _{n \rightarrow \infty} \sum_{i=1}^{n} M\left(\Delta_{i}^{\prime}, \Delta_{i}^{\prime}\right)+\lim _{n \rightarrow \infty} \sum_{i \neq j}^{n} M\left(\Delta_{i}^{\prime}, \Delta_{j}^{\prime}\right) .
\end{aligned}
$$

By the nonnegativity of $M\left(\Delta_{i}, \Delta_{i}\right)$ and the remark above we have that $\lim _{n \rightarrow \infty} M\left(\delta_{n}, \delta_{n}\right)$ exists and is finite. We must establish that $M(\Delta, \Delta)$ is well defined. So, suppose also that $\Delta=\lim _{n \rightarrow \infty} \rho_{n}$, where $\rho_{n}=\sum_{i=1}^{n} \Delta_{i}^{\prime \prime}$, and that each $\Delta_{i}^{\prime} \subset \Delta_{j}^{\prime \prime}$, for some $j$. Then the limits are clearly the same, that is

$$
\lim _{n \rightarrow \infty} M\left(\delta_{n}, \delta_{n}\right)=\lim _{n \rightarrow \infty} M\left(\rho_{n}, \rho_{n}\right) .
$$

Now suppose $\zeta_{n}=\sum_{i=1}^{n} \Delta_{i}^{\prime \prime \prime}$ gives a third representation of $\Delta$, as $n \rightarrow \infty$. Let $\rho_{n}$ be defined in such a way that each $\Delta_{i}^{\prime \prime} \subset \Delta_{j}^{\prime}$ for some $j$ and $\Delta_{i}^{\prime \prime} \subset \Delta_{i}^{\prime \prime \prime}$ for some $k$. It is now easy to see that

$$
\lim M\left(\zeta_{n}, \zeta_{n}\right)=\lim M\left(\delta_{n}, \delta_{n}\right) ;
$$

thus $M$ is well defined.

If $\Delta \subset \Delta^{\prime}$, define

$$
M\left(\Delta, \Delta^{\prime}\right)=M(\Delta, \Delta)+M\left(\Delta, \Delta^{\prime}-\Delta\right) .
$$

Finally if $\Delta=\Delta_{1}+\Delta_{2}$, we have

$$
M(\Delta, \Delta)=M\left(\Delta_{1}, \Delta_{1}\right)+M\left(\Delta_{2}, \Delta_{2}\right)+2 \operatorname{Re} M\left(\Delta_{1}, \Delta_{2}\right),
$$

by use of the usual interior limits, the continuity of $K$, the zero PD condition, and Lemma 3.1. Thus, $M\left(\Delta, \Delta^{\prime}\right)$ is now completely defined and is continuous by the zero PD condition. It remains to show that $M$ is of the nonnegative type. By construction, $M$ is positive semidefinite for any partition having binary points of division. Now let $\Xi=\Delta_{1}, \ldots, \Delta_{n}$ be any partition. Then there is a partition $\beth^{\prime}$ with binary points of division, $\delta_{1}, \delta_{2}, \ldots, \delta_{2 n-1}$, where $\delta_{2 i-1}, i=1, \ldots, n$, is interior and a close approximation to $\Delta_{i}$, and $\delta_{2 i}$ is the gap between $\delta_{2 i-1}$ and $\delta_{2 i+1}$. Now consider any sequence $\rho_{1}, \ldots, \rho_{2 n-1}$ of complex numbers, with $\rho_{2 i}=0$ for each $i$. Then 


$$
\sum M\left(\delta_{i}, \delta_{j}\right) \rho_{i} \bar{\rho}_{j}=\sum M\left(\delta_{2 i-1}, \delta_{2 j-1}\right) \rho_{2 i-1} \bar{\rho}_{2 j-1} \geq 0
$$

If $\beth_{m}^{\prime}$ is a sequence of partitions of $2 n-1$ elements, $\delta_{1}^{m}, \ldots, \delta_{2 n-1}^{m}$, with $\delta_{2 i-1}^{m} \subset \Delta_{i}$ and $\lim \left|\delta_{2 i}^{m}\right|=0$ and $\lim \delta_{2 i-1}^{m}=\Delta_{i}$, we see that

$$
\lim _{m \rightarrow \infty} M\left(\delta_{i}^{m}, \delta_{j}^{m}\right) \rho_{i} \rho_{j}=\sum M\left(\Delta_{i}, \Delta_{j}\right) \rho_{2 i-1} \bar{\rho}_{2 j-1} \geq 0
$$

Thus $M$ is of the nonnegative type, and the theorem is proved.

Referring back to the proof of Theorem 3.5 we prove the

Corollary 3.6. $\lim _{n \rightarrow \infty} \sum_{i} M\left(\Delta_{i}^{n}, \Delta_{i}^{n}\right)=\lim _{n \rightarrow \infty}\left(\operatorname{tr} A_{n}\right)$.

Proof. Since $\operatorname{tr} A_{n} \geq \operatorname{tr} A_{n-1}$, the limit on the right exists. Now for any $n$ and $\varepsilon>0$ there is an $M$ such that for $m>M$

$$
\left|\sum_{i} M\left(\Delta_{i}^{n}, \Delta_{i}^{n}\right)-\sum_{i} \sum_{\Delta_{j}^{m} \subset \Delta_{i}^{n}} K_{m}\left(\Delta_{j}^{m}, \Delta_{j}^{m}\right)+\sum_{i} \sum_{\Delta_{k}^{m}, \Delta_{j}^{m} \subset \Delta_{i}^{n} ; j \neq k} K_{m}\left(\Delta_{j}^{m}, \Delta_{k}^{m}\right)\right|<\varepsilon
$$

Now by Lemma 3.1 the rightmost term on the left side of (3.7) tends to zero as $n$ increases, and the middle term is $\operatorname{tr} A_{m}$. Taking the limit as $n$ tends to $\infty$ gives the result.

We say that a covariance function $N\left(\Delta, \Delta^{\prime}\right)$ is minimal for its cross covariance if for any covariance function $L\left(\Delta, \Delta^{\prime}\right)$ arising from a process with orthogonal increments the interval function $N\left(\Delta, \Delta^{\prime}\right)-L\left(\Delta, \Delta^{\prime}\right)$ is not a covariance. With Corollary 3.6 we can prove the

Corollary 3.8. The covariance function $M(\Delta, \Delta)$ is minimal.

Proof. Let $L\left(\Delta, \Delta^{\prime}\right)$ be the covariance of a process having orthogonal increments, and define $M^{\prime}(\Delta, \Delta)=M\left(\Delta, \Delta^{\prime}\right)-L\left(\Delta, \Delta^{\prime}\right)$. For $n$ large enough we have, by (3.7),

$$
\sum M^{\prime}\left(\Delta_{i}^{n}, \Delta_{i}^{n}\right) \leq \operatorname{tr} A_{m}+\delta_{m}+\varepsilon-L\left(\Delta_{1}^{0}\right)
$$

where $\delta_{m}$ is as small as desired. Then the trace of $M^{\prime}$ is not large enough to support its cross covariance because $\operatorname{tr}\left(A_{m}\right)$ is minimal. This gives the result.

Now let $X(t)$ be any process of bounded quadratic variation with quadratic variation satisfying (w1) and (w2) conditions. Then by Lemma 3.3 the covariance function of $X, K\left(\Delta, \Delta^{\prime}\right)$, satisfies a zero PD condition. Hence applying Theorem 3.5 and Corollary 3.8 we obtain a minimal covariance function $M\left(\Delta, \Delta^{\prime}\right)$ supporting the cross covariance of $K$. Taking the difference $K\left(\Delta, \Delta^{\prime}\right)-M\left(\Delta, \Delta^{\prime}\right)$ we get a nonnegative interval function $L\left(\Delta, \Delta^{\prime}\right)$ representing a process with orthogonal increments. We summarize this in the

Theorem 3.9. If $X(t)$ is a stochastic process of bounded quadratic variation with quadratic measure satisfying (w1) and (w2) conditons, its covariance can be written 
as a sum of two covariance functions, $L\left(\Delta, \Delta^{\prime}\right)$ and $M\left(\Delta, \Delta^{\prime}\right)$, where $L\left(\Delta, \Delta^{\prime}\right)$ is the covariance of a process having orthogonal increments, and $M\left(\Delta, \Delta^{\prime}\right)$ is the minimal covariance supporting the cross covariance of $X(t)$.

This theorem applies to the continuous process in $\operatorname{Lip}\left(\phi_{1}, \phi_{2}, \not\right)$ where $\phi_{1}$ and $\phi_{2}$ are self pairable, for which Young has defined stochastic integrals [4].

\section{REFERENCES}

1. J. L. Doob, Stochastic processes, Wiley, New York; Chapman \& Hall, London, 1953. MR 15, 445.

2. Paul Lévy, Processus stochastiques et mouvement brownien, 2ième éd., Gauthier-Villars, Paris, 1965. MR 32 \# 8363.

3. P. Mesani, Orthogonally scattered measures, Advances in Math., vol. 2, Princeton Univ. Press, Princeton, N.J., 1968, pp. 61-117.

4. L. C. Young, Some new stochastic integrals. I. Analogues of Hardy-Littlewood classes, Advances in Probability 2 (1970), 163-240.

Department of Mathematics, Texas A\&M University, College Station, Texas 77843 